\title{
Comparison of two Meta-Heuristics for the Bi- Objective Flexible Job Shop Scheduling Problem with Sequence Dependent Setup Times
}

\author{
João Sacramento $^{\mathrm{a}}$, João Pedrosa ${ }^{\mathrm{a}}$, Nelson Chibeles-Martins ${ }^{\mathrm{b}}$ *, Tânia Pinto- \\ Varela $^{\mathrm{a}}$ \\ ${ }^{a}$ Centro Estudos Gestão, Instituto Superior Tecnico, Universidade Lisboa, Lisboa, \\ Portugal \\ ${ }^{b}$ Centro de Matemática e Aplicações and Departamento de Matemática, Faculdade de \\ Ciências e Tecnologia, UNL, 2829-516 Caparica, Portugal \\ npm@fct.unl.pt
}

\begin{abstract}
The increasingly competitivity in the plastic container market is driving companies toward a greater focus on efficiency, and mass production customisation, which triggers the increase of productivity by implementing more efficient and faster IT solutions. This work is based on a Portuguese case study, to develop a scheduling model considering the specific characteristics of this type of facilities and increase its competitiveness. To this end, two different approaches, the Tabu Search and Genetic Algorithm, were developed to solve a flexible job shop scheduling problem under a make-to-order production strategy. Each approach was validated using the case study, and the model's applicability were testes trough five instances. The results have shown that Tabu Search has a better efficacy and the Genetic Algorithm shows better efficiency.
\end{abstract}

Keywords: Flexible Job Shop Problem, Tabu Search, Genetic Algorithm, Metaheuristics, Multi-objective

\section{Introduction}

Nowadays, the industrial sector faces a new challenge. The traditional tactics used to increase productivity are outdated and companies are looking for new factors that may lead to improvement. Considering the fourth industrial revolution, Industry 4.0, more detailed and deep problem characterization is necessary. The speed of sharing information to and from the facility is crucial. The real-time information availability is required to support the decision making. To satisfy this challenge an agile and fast solution approach, with the aim of increasing flexibility and get competitive leverage is necessary. Therefore companies are becoming highly invested in developing customized information systems to manage, plan and schedule its manufacturing processes.

Despite some research has already been made using exact approaches, the FJSP's complexity and intractability usually lead to high computational times when these kind of algorithms are applied. On the other hand, meta-heuristics go towards the fourth revolution concepts, with lower computational burdens, being the Tabu Search (TS) and Generic Algorithm (GA), two of the most promising alternatives. Therefore the authors opted for focusing their efforts on the proposed Metaheuristics approaches. 
Some of the work developed using a mono-objective TS were developed by Dell'Amico and Trubian (1993) with an algorithm that solve the classical Job-Shop Scheduling Problem (JSSP) makespan minimization. However, several authors, like Brandimarte (1993), Dauzère-pérès and Paulli (1997), Mastrolilli and Gambardella (2000), C. Scrich, V. Armentano (2004), and Abdelmaguid (2015) developed TS to solve the Flexible JobShop Scheduling Problem (FJSSP) in regards to makespan minimization, except C. Scrich, V. Armentano (2004) whom proposed to minimize tardiness. More recently the authors Saidi-Mehrabad and Fattahi (2007) and Shen et al. (2018) considered the sequence dependent setup times case of the FJSSP. GA approaches have also been applied to Scheduling problems. Pezzella et al. (2008) proposed several improvements to the generic GA in order to make them more efficient solving SP.

However, some research has been done concerning multi-objective approaches. Jia and $\mathrm{Hu}$ (2014) developed a TS procedure based on path relinking to solve a multi-objective FJSSP considering the minimization of the makespan, total workload and maximum workload, using the Pareto approach. Li et al. (2010) proposed a hybrid TS based algorithm to solve the multi-objective FJSSP with the three criteria chosen being makespan, total workload and maximum workload. Murata and Ishibuchi (1995) proposed a framework for GA with a weighted sum using randomly specified weights for each selection. It was already applied to several multi-objective optimization problems but never to a FJSSP. Morinaga et al. (2014) solved a FJSSP with MTO policy using a GA to minimize tardiness and setup-worker load. It was used a classic weighted sum approach to deal with the bi-objective problem.

The proposed work explores two multi-objective approach, a Genetic and a Tabu Search algorithm to define the production scheduling considering the tardiness and makespan minimization, as objective functions. The facility follows a make-to-order strategy, with sequence dependent setup time. To illustrate the methodologies applicability and its performance, not only a real case study is explored, but also, five more instances. This work is based on the same case studied in Chibeles-Martins et al. (2017).

\section{Modelling Characterization}

Multi-objective Genetic Algorithm - BObGA

A new multi-objective genetic algorithm is developed based on the classical algorithm proposed by the authors Murata and Ishibuchi in (1995) extending it with the integration of randomly weights for each selection of the best chromosomes. To take into account the mitigation of customers' service level impact, the tardiness has a higher importance than the makespan. This is achieved by introducing a new parameter defining a lower bound that controls the minimum weight that is applied to the tardiness. Finally, the BObGA uses a hill climbing heuristic introduced by Greiner (1992) to perform a local search. The BObGA algorithm pseudocode is shown in Figure 1.

\section{Multi-objective Tabu Search Algorithm - BObTS}

The Tabu Search proposed algorithm is based on Shen et al. in (2018) extended to explore a Lexicographic Multi-objective approach, considering the tardiness minimization, with highest priority and the makespan minimization as a second priority objective. It uses the neighborhood structure developed on the aforementioned work but is adapted to be applicable in a multi-objective perspective by assigning different importance levels to objective functions according to the stage the algorithm is in. A diversification strategy is employed to avoid a premature stop at local minima. The BObTS algorithm pseudocode is presented in Figure 2. 


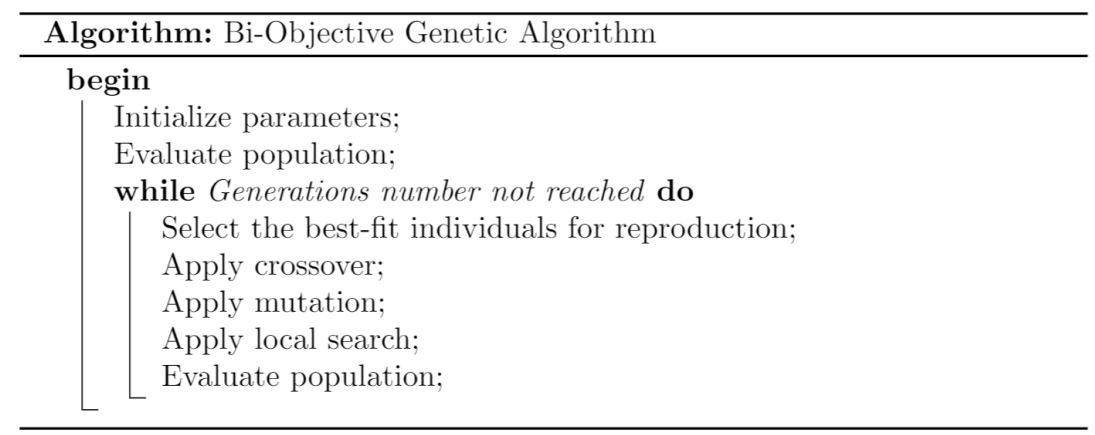

Figure 1-Bi-objective Genetic algorithm pseudocode - BobGA

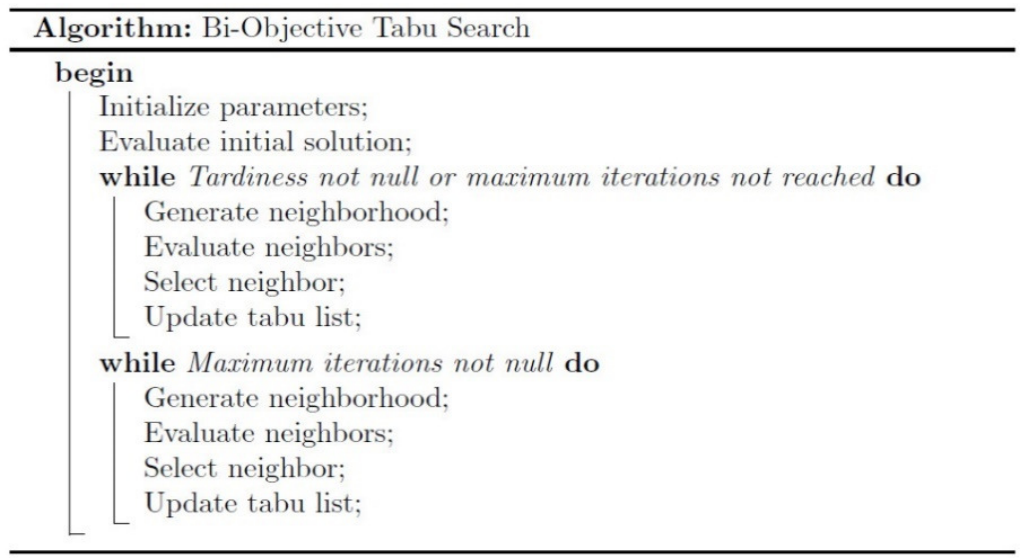

Figure 2 - Bi-objective Tabu Seach pseudocode - BObTS.

\section{Instances Characterization}

The case study (CS) is based on a mould industry production of plastics containers. The plastic containers are characterized by a bottom and a cover, produced using an injection moulding process. The process requires different changeovers, based on the product sequence production, each one with different setup time associated. The production process requires mainly three tasks. However, each product manufacture follows a different path, triggering different setup times. Nevertheless, all machines are able to produce all the products. For confidential reasons detailed production process information is omitted. Beyond the case study implementation, five instances with increase complexity were explored over the two approaches. In Table 1 is shown instances' characterization with the respective number of orders, the produced quantity, due date and number of machines. 
Table 1 - Instances characterization.

\begin{tabular}{ccccc}
\hline Instances & \#orders & Quantity (units) & $\begin{array}{c}\text { Average due } \\
\text { date (minutes) }\end{array}$ & \# Machines \\
\hline CS & 40 & 8888 & 2200 & 8 \\
$\mathbf{1}$ & 70 & 2162 & 1765 & 8 \\
$\mathbf{2}$ & 30 & 9797 & 853 & 8 \\
$\mathbf{3}$ & 40 & 5000 & 880 & 8 \\
$\mathbf{4}$ & 40 & 8888 & 1765 & 7 \\
$\mathbf{5}$ & 100 & 7189 & 2091 & 8 \\
\hline
\end{tabular}

\section{Results}

BObGA and BObTS algorithms were executed for the six instances on a PC with an Intel Core i7-6700HQ, 2.60GHz, 8 GB RAM. A detailed characterization of the results obtained are shown in Table 2, and its graphic representation in

Figure 4.

Figure 3 and

Table 2 - BObGA and BObTS performance results.

\begin{tabular}{|c|c|c|c|c|c|c|}
\hline & \multirow[b]{2}{*}{ Instance } & \multirow[b]{2}{*}{$\begin{array}{c}\text { (Order x } \\
\text { Machine) }\end{array}$} & \multicolumn{2}{|c|}{ Tardiness } & \multirow{2}{*}{$\begin{array}{c}\text { Makespan } \\
\begin{array}{c}\text { Average } \\
\text { (min) }\end{array}\end{array}$} & \multirow{2}{*}{$\begin{array}{c}\text { CPU Time } \\
\begin{array}{c}\text { Average } \\
\text { (seconds) }\end{array}\end{array}$} \\
\hline & & & $\begin{array}{c}\% \\
\text { Optimal } \\
\text { values }\end{array}$ & $\begin{array}{c}\text { Average } \\
\text { (min) }\end{array}$ & & \\
\hline \multirow{6}{*}{ 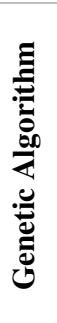 } & $\mathrm{CS}$ & $40 \times 8$ & $84 \%$ & 8.6 & $1,510.2$ & 27.7 \\
\hline & 1 & $70 \times 8$ & $98 \%$ & 0.2 & $1,160.1$ & 143.1 \\
\hline & 2 & $30 \times 8$ & $86 \%$ & 19,4 & $1,018.2$ & 17.1 \\
\hline & 3 & $40 \times 8$ & $48 \%$ & 46 & $1,040.3$ & 32.1 \\
\hline & 4 & $40 \times 7$ & $48 \%$ & 130.3 & 1,950 & 34.9 \\
\hline & 5 & $100 \times 8$ & $0 \%$ & 718.4 & $2,892.5$ & 364.3 \\
\hline \multirow{6}{*}{ 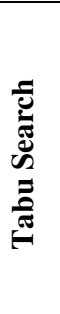 } & CS & $40 \times 8$ & $100 \%$ & 0 & $1,349.5$ & 267 \\
\hline & 1 & $70 \times 8$ & $100 \%$ & 0 & 994.4 & 300 \\
\hline & 2 & $30 \times 8$ & $100 \%$ & 0 & 887.3 & 301 \\
\hline & 3 & $40 \times 8$ & $87 \%$ & 4 & 931.3 & 304 \\
\hline & 4 & $40 \times 7$ & $0 \%$ & 395 & $1,833.3$ & 303 \\
\hline & 5 & $100 \times 8$ & $0 \%$ & 425.5 & $2,910.4$ & 663 \\
\hline
\end{tabular}

The BObTS reaches the optimum value for the tardiness objective function (which is zero value) in all iteration, in the Instances: CS, 1 and 2. In third Instance $87 \%$ of iterations reached the optimal value, followed by instances 4 and 5 , with a nonoptimum, justified by the decreased number of machines or the increase number of orders. The BObGA shown a decrease number of optimal solutions for the tardiness, as the complexity increases, with Instance 5, reaching only non-optimum solution.

However, considering the case study, BObTS showed a $100 \%$ of optimum solutions for the Tardiness vs the $84 \%$ for the BObGA (Table 2). As is shown in 
Figure 4, the BObGA is more efficient, being less time consuming than BObTS.

However, BObTS denotes a higher efficacy (

Figure 3).

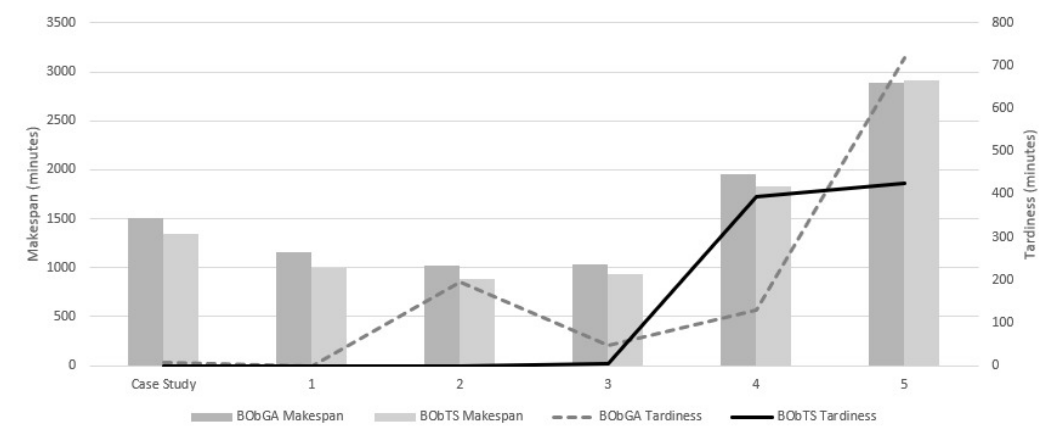

Figure 3 - BObGA and BObTS non dominated solution characterization

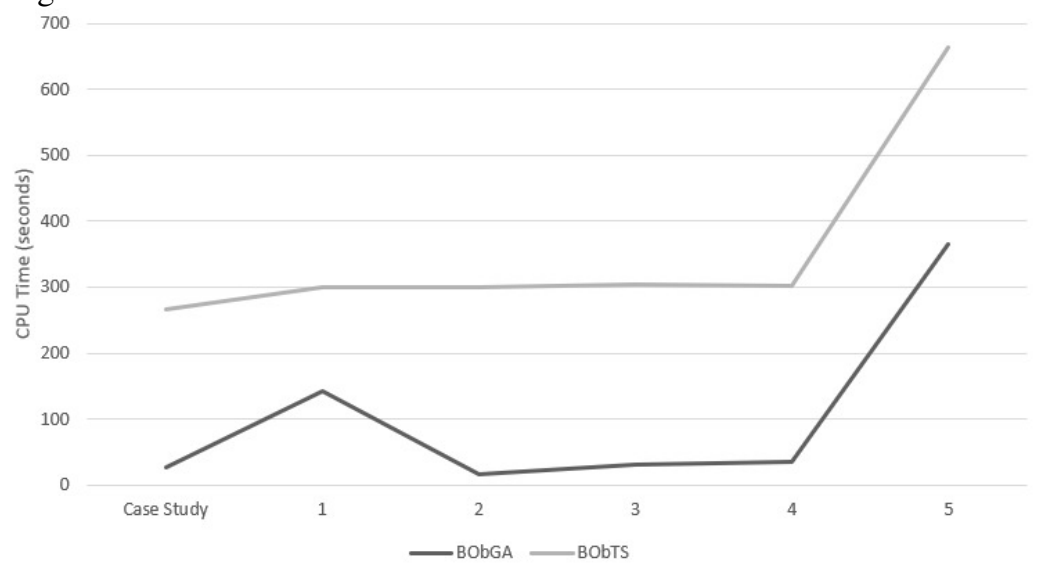

Figure 4 - BObGA and BObTS computational performance.

\section{Conclusion}

Nowadays, industry is triggered to increase its efficiency and productivity by developing tools to support decision making processes in real time. To overcome this challenge the authors propose two Meta-heuristic multi-objective algorithms (BObGA and BObTS) to explore the scheduling of a flexible job shop facility with product sequence setups. Both Meta-heuristics considered the tardiness and makespan minimization as objective functions. The MObGA explored the randomly weighted sum, while the MObTS a lexicographic approach. The Algorithm applicability and its performance comparison were made over a real case study, followed by five instances with increased complexity, to stress the algorithms. As the results showed, BObTS algorithm outperformed the BObGA in almost all cases, reaching better objective functions values denoting a higher efficacy. However, BObGA is less time consuming showing a higher efficiency. Nevertheless, further work should be done, exploring other production strategies and stressing the algorithm with tighter due dates. 


\section{Acknowledgements}

The authors gratefully acknowledge the support of the Portuguese National Science Foundation through Portugal 2020 project POCI-01-0145-FEDER-016418 by UE/FEDER through the program COMPETE2020. This work was partially supported by the Fundação para a Ciência e a Tecnologia (Portuguese Foundation for Science and Technology) through the project UIDB/00297/2020 (Centro de Matemática e Aplicações).

\section{References}

T. F. Abdelmaguid, 2015, A neighborhood search function for flexible job shop scheduling with separable sequence-dependent setup times. Applied Mathematics and Computation, 260, 188-203. https://doi.org/10.1016/J.AMC.2015.03.059

P. Brandimarte, 1993, Routing and scheduling in a flexible job shop by tabu search. Annals of Operations Research, 41(3), 157-183. http://doi.org/10.1007/BF02023073

N. Chibeles-Martins, A. Marques, and T. Pinto-Varela, 2017, A Bi-objective two step Simulated Annealing Algorithm for Production Scheduling. Computer Aided Chemical Engineering, 40, 1351-1356. https://doi.org/10.1016/B978-0-444-639653.50227-0

C. R. Scrich, V. A. Armentano and M. Laguna, 2004, Tardiness minimization in a Flexible job shop : A tabu search approach, Journal of Intelligent Manufacturing, 15, pp. 103-115, http://dx.doi.org/10.1023/B:JIMS.0000010078.30713.e9

S. Dauzère-Pérès and J. Paulli, 1997, An integrated approach for modeling and solving the general multiprocessor job-shop scheduling problem using tabu search. Annals of Operations Research, 70(0), 281-306. https://doi.org/10.1023/A:1018930406487

M. Dell'Amico and M. Trubian, 1993, Applying tabu search to the job-shop scheduling problem. Annals of Operations Research, 41(3), 231-252.

R. Greiner, 1992, Probabilistic Hill-Climbing: Theory and Applications. Proceedings of the Ninth Canadian Conference on Artificial Intelligence.

S. Jia and Z. Hu, 2014, Path-relinking Tabu search for the multi-objective flexible job shop scheduling problem', Computers and Operation Research. Elsevier, 47, pp. 1126. DOI: 10.1016/j.cor.2014.01.010

J.-Q. Li, Q.-K. Pan and Y.-C. Liang, 2010, An effective hybrid tabu search algorithm for multi-objective flexible job- shop scheduling problems, Computers \& Industrial Engineering, 59(4), pp. 647-662. https://doi.org/10.1016/j.cie.2010.07.014

M. Mastrolilli and L. M. Gambardella, 2000, Effective neighbourhood functions for the flexible job shop problem. Journal of Scheduling, 3(1), 3-20.

Y. Morinaga, M. Nagao and M. Sano, 2014, Optimization of flexible job-shop scheduling with weighted tardiness and setup-worker load balance in make-to-order manufacturing. In Joint 7th International Conference on Soft Computing and Intelligent Systems (SCIS) and 15th International Symposium on Advanced Intelligent Systems (ISIS) (pp. 87-94). https://doi.org/10.1109/SCIS-ISIS.2014.7044681

T. Murata and H. Ishibuchi, 1995, MOGA: Multi-objective genetic algorithms. In Proceedings of 1995 IEEE International Conference on Evolutionary Computation (Vol. 1, pp. 289-). https://doi.org/10.1109/ICEC.1995.489161

F. Pezzella, G. Morganti and G. Ciaschetti, 2008, A genetic algorithm for the Flexible Job-shop Scheduling Problem. Computers and Operations Research, 35(10), 32023212. https://doi.org/10.1016/j.cor.2007.02.014. 
Comparison of two Meta-Heuristics for the Bi-Objective Flexible Job Shop Scheduling Problem with Sequence Dependent Setup Times

M. Saidi-Mehrabad and P. Fattahi, 2007, Flexible job shop scheduling with tabu search algorithms. The International Journal of Advanced Manufacturing Technology, 32(5), 563-570. https://doi.org/10.1007/s00170-005-0375-4.

L. Shen, S. Dauzère-Pérès and J. S. Neufeld, 2018, Solving the flexible job shop scheduling problem with sequence-dependent setup times, European Journal of Operational Research, 265(2), 503-516. DOI: 10.1016/j.ejor.2017.08.021 